\title{
Generalised Inverse Mellin Invariant Transform
}

Benedict Irwin ${ }^{1}$

${ }^{1}$ University of Cambridge

January 7, 2021

In a previous article [1], we found a specific integral transform $\mathcal{Q}$, such that

$$
\mathcal{Q}\left[\mathcal{M}^{-1}[f(s)](x)\right](t)=\mathcal{M}^{-1}[f(s)](t)
$$

for the case that $f(s)=\Gamma(s) \zeta(s)$, as this had a functional equation which could be used to define an invariance. In this work we create a generalised version of this.

If we have a general functional relationship for $f(x)$

$$
f(s)=h(s) f(g(s))
$$

where $g(s)$ has a nice inverse $g^{-1}(s)$.

Then the prescription for getting a $\mathcal{Q}$ that meets the requirement of equation 1 is finding a kernel to define the transform $\mathcal{Q}$ for an inverse power as

$$
\mathcal{Q}\left[x^{-s}\right](q, s)=\int_{0}^{\infty} x^{-s} k(x, q) d x=q^{-g^{-1}(s)} \frac{h\left(g^{-1}(s)\right)}{g^{\prime}\left(g^{-1}(s)\right)}
$$

Thus for a test function $\phi(s)$,

$$
\begin{gathered}
\mathcal{Q}\left[\mathcal{M}^{-1}[\phi]\right]=\mathcal{Q}\left[\frac{1}{2 \pi i} \int_{c-i \infty}^{c+i \infty} x^{-s} \phi(s) d s\right] \\
\mathcal{Q}\left[\mathcal{M}^{-1}[\phi]\right]=\frac{1}{2 \pi i} \int_{c-i \infty}^{c+i \infty} \mathcal{Q}\left[x^{-s}\right] \phi(s) d s \\
\mathcal{Q}\left[\mathcal{M}^{-1}[\phi]\right]=\frac{1}{2 \pi i} \int_{c-i \infty}^{c+i \infty} q^{-g^{-1}(s)} \frac{h\left(g^{-1}(s)\right)}{g^{\prime}\left(g^{-1}(s)\right)} \phi(s) d s
\end{gathered}
$$

by letting $s \rightarrow g(t)$ we get

$$
\mathcal{Q}\left[\mathcal{M}^{-1}[\phi]\right]=\frac{1}{2 \pi i} \int_{g^{-1}(c-i \infty)}^{g^{-1}(c+i \infty)} q^{-t} h(t) \phi(g(t)) d t=\mathcal{M}^{-1}[h(t) \phi(g(t))]
$$

the condition for this to remain an inverse Mellin transform (up to sign) is that $g^{-1}(c+i \infty) \rightarrow d \pm i \infty$ and $g^{-1}(c-i \infty) \rightarrow d \mp i \infty$. When $\phi(s)=f(s)$ which satisfies the functional equation, then equation 1 is satisfied. 


\section{Getting the Kernel Function}

Because we have chosen our transform to be the inverse Mellin transform, we can extract our kernel function by taking the inverse Mellin transform of the desired result of $\mathcal{Q}\left[x^{-s}\right]$.

Then the kernel function should be given by

$$
k(q, x)=\mathcal{M}^{-1}\left[q^{-s} \frac{h(s)}{g^{\prime}(s)}\right](x)
$$

\section{Example}

Take the Riemann zeta function again, but this time without the additional gamma function

$$
\zeta(s)=2^{s} \pi^{s-1} \sin \left(\frac{\pi s}{2}\right) \Gamma(1-s) \zeta(1-s)
$$

Here we have

$$
h(s)=2^{s} \pi^{s-1} \sin \left(\frac{\pi s}{2}\right) \Gamma(1-s) g(s)=1-s g^{-1}(s)=1-s g^{\prime}(x)=-1 g^{-1}(c \pm i \infty)=d \mp i \infty
$$

we may need to include an additional negative factor due to the integral limits swapping sign.

We assemble

$$
k_{f}(q, x)=\mathcal{M}^{-1}\left[-q^{-s} 2^{s} \pi^{s-1} \sin \left(\frac{\pi s}{2}\right) \Gamma(1-s)\right](x)=\frac{2 \cos \left(\frac{2 \pi}{q x}\right)}{q x}
$$

If we take the modular form

$$
y(z)=y\left(\frac{2 z+3}{z+2}\right)
$$

then

$$
h(s)=1 g(s)=\frac{2 s+3}{s+2} g^{-1}(s)=\frac{3-2 s}{s-2} g^{\prime}(s)=\frac{1}{(s+2)^{2}}
$$

there is a sign swap

$$
k_{y}(q, x)=\mathcal{M}^{-1}\left[q^{-s}(s+2)^{2}\right](x)
$$

this doesn't work so well.

Starting from a simpler example

$$
f(x)=\Gamma(x) f(1-x)
$$

gives $k(x, q)=-e^{-q x}$, then

$$
\mathcal{Q}\left[x^{-s}\right]=-q^{s-1} \Gamma(1-s)
$$

which apparently is connected to the Laplace Transform. Likewise

$$
f(s)=(-i)^{s} \Gamma(s) f(1-s)
$$

gives the kernel $-e^{-i q x}$ which will relate to the Fourier transform.

For the functional equation

$$
f(s)=\Gamma(s) f\left(\frac{s^{2}}{2}\right)
$$

we get

$$
k(q, x)=\mathcal{M}^{-1}\left[q^{-s} s^{-1} \Gamma(s)\right]=\Gamma(0, q x)
$$


This defines a transform such that

$$
\mathcal{Q}\left[x^{n}\right]=\frac{\Gamma(n+1)}{(n+1) q^{n+1}}, q>0
$$

which seems quite fundamental in terms of differentiation. Another interesting one satisfies

$$
f(s)=\Gamma(s) f\left(s+s^{2}\right)
$$

which gives

$$
k(q, x)=e^{-q x}-\sqrt{\pi q x}+\sqrt{\pi q x} \operatorname{erf}(\sqrt{q x})
$$

and

$$
\begin{gathered}
f(s)=\Gamma(s) f\left(s+\frac{s^{2}}{2}\right) \\
k(q, x)=e^{-q x}-q x \Gamma(0, q x)
\end{gathered}
$$

for which

$$
\mathcal{Q}\left[x^{n}\right]=\frac{\Gamma(n+1)}{(n+2) q^{n+1}}, q>0
$$

\section{References}

[1] - Riemann Zeta Invariance Under Composed Integral Transform, https://www.authorea.com/users/5445/articles/436204-riemann-zeta-invariance-under-composed-integraltransform 